Case Report

\title{
Clinical Impact of Consolidative and Salvage Radiotherapy for Lymph Node Metastasis in Upper Urinary Tract Urothelial Carcinoma
}

\author{
Hideyuki Kondo, Suguru Shirotake (D), Takashi Okabe, Soichi Makino, \\ Koshiro Nishimoto $\mathbb{D}$, and Masafumi Oyama \\ Department of Uro-Oncology, Saitama Medical University International Medical Center, Saitama, Japan \\ Correspondence should be addressed to Suguru Shirotake; ss197816@saitama-med.ac.jp \\ and Koshiro Nishimoto; k.nishimoto@keio.jp
}

Received 22 November 2017; Accepted 14 March 2018; Published 22 April 2018

Academic Editor: Sigurdur Gudjonsson

Copyright (C) 2018 Hideyuki Kondo et al. This is an open access article distributed under the Creative Commons Attribution License, which permits unrestricted use, distribution, and reproduction in any medium, provided the original work is properly cited.

A 75-year-old Japanese male was referred to our institution for the evaluation of a left ureteral tumor in the ureterovesical junction. Computed tomography and pathologic examination under ureteroscopy revealed an invasive left ureteral urothelial carcinoma with left obturator nodal metastasis without distant metastasis. First, the patient underwent systemic chemotherapy (gemcitabine and cisplatin chemotherapy). We then performed left radical nephroureterectomy and extended lymph node dissection. Pathological examination revealed that the tumor was a high-grade invasive urothelial carcinoma with left common iliac and pelvic lymph node metastasis (pT3N2). Unfortunately, metastases appeared in the common iliac and para-aortic lymph nodes immediately after the operation; therefore, the previous first-line chemotherapy was readministered and second-line chemotherapy (gemcitabine and paclitaxel chemotherapy) was also performed. We also performed consolidative radiotherapy and salvage radiotherapy (boost, $20 \mathrm{~Gy} / 10$ fractions to the inferior para-aortic, and left common iliac regions containing swollen lymph nodes). The patient has shown no evidence of recurrence or metastasis even approximately 4 years after the initial diagnosis of advanced UUT-UC with lymph node metastasis. Our case suggests that consolidative or salvage radiotherapy combined with surgery and chemotherapy may provide clinical benefit for selected cases of advanced UUT-UC with lymph node metastasis.

\section{Introduction}

Upper urinary tract urothelial carcinoma (UUT-UC) is relatively rare and accounts for $5-10 \%$ of urothelial malignancies [1]. Generally, the 5-year survival rates of patients with pTa or pT1 UUT-UC (Stage 0-I in the AJCC staging system) are over $60 \%$, whereas the rate for patients with pT3-4 or N1-3 disease (Stage IV) is extremely poor $(<5 \%)$ [2]. According to a report of a large series of patients with UUT-UC treated with radical nephroureterectomy (RNU), the 5-year recurrencefree survival rates of pT4 and N1-3 diseases were only $4.7 \%$ and $29 \%$, respectively, and therefore the application of multimodality therapy for Stage IV UUT-UC disease may be considered. Herein, we report a case of Stage IV UUT-UC that was successfully treated with RNU, chemotherapy, and radiotherapy.

\section{Case Description}

A 75-year-old Japanese male, who was admitted to another hospital with renal dysfunction and microscopic hematuria at a medical checkup, was diagnosed with a left lower ureteral tumor. The patient was referred to the Saitama Medical University International Medical Center for treatment of the tumor. As for the relevant past history, the patient had received stent-graft implantation for the treatment of abdominal aortic aneurism. There was no significant family history. Physical examination revealed normal appearance except for high body mass index $\left(27.3 \mathrm{~kg} / \mathrm{m}^{2}\right.$ [normal range $<25 \mathrm{~kg} / \mathrm{m}^{2}$ ], body weight: $78.4 \mathrm{~kg}$, height: $1.69 \mathrm{~m})$. Laboratory data were normal except for serum creatinine (1.56 [normal range: $0.43-1.08] \mathrm{mg} / \mathrm{dl}$ ) and creatinine clearance $(50.2 \mathrm{ml} / \mathrm{min})$. Urine cytological examination detected urothelial carcinoma 
cells. Magnetic resonance imaging suggested the ureteral tumor $(21 \times 13 \mathrm{~mm})$ had invaded the muscle layer, but not the periureteric fat (Figure S1). Computed tomography (CT) showed a contrast-enhanced tumor in the lower ureter (data not shown), left hydronephrosis (data not shown) and an enlarged left obturator lymph node $(18 \mathrm{~mm}$ in diameter, Figure S2). There was no significant finding in the bladder using cystoscopy. By tumor biopsy using a ureteroscope, the tumor was pathologically diagnosed as a high-grade urothelial carcinoma (UC, clinical stage: cT2, N1, M0). The patient underwent several modality therapies (Table S1). The treatment efficacy was evaluated by CT imaging (Figure S2), in which the sizes of the LNs were retrospectively measured by HK, SS, TO, and AK (Table S1) and compared by statistical analysis (Student's $t$-test). In the comparative analysis, a $p$ value below 0.05 was considered significant.

In order to control the apparent obturator LN metastasis $(17.5 \pm 0.5 \mathrm{~mm}$, Figure 1(a), Table S1), the patient was given 6 courses of chemotherapy with gemcitabine $\left(1,000 \mathrm{mg} / \mathrm{m}^{2}\right.$, days 1,8 , and 15$)$ and cisplatin $\left(70 \mathrm{mg} / \mathrm{m}^{2}\right.$, day 2$)$ (GC-chemotherapy, GC \#1-6) [3, 4]. Other LNs, which subsequently showed enlargement, were within normal size $(<1.0 \mathrm{~cm})$ before GC-chemotherapy (Figures 1(b)-1(d)). Although the obturator LN significantly shrunk to $8.4 \pm 1.1 \mathrm{~mm}$ (Figure 1(e)), the left common iliac LN enlarged from $6.6 \pm 0.4$ to $11.0 \pm 0.4 \mathrm{~mm}$ (Figure 1(f)). The two para-aortic LNs, which later expanded, remained within the normal size range $(6.4 \pm$ 0.3 and $7.1 \pm 0.8 \mathrm{~mm}$, resp., Figures $1(\mathrm{~g})-1(\mathrm{~h})$ ).

In order to treat the ureteral tumor and common iliac LN metastasis, in which cancer cells may acquire resistance to GC-chemotherapy, the patient underwent left RNU and lymph node dissection (LND), including most of the common iliac LNs. Simultaneously, regional external iliac, obturator, and internal iliac LNs were removed as previously reported $[5,6]$. However, we could not remove the enlarged LN behind the common iliac artery (* in Figure 1(f)) owing to vascular adherence, probably caused by stent-graft implantation for the treatment of aortic aneurism (Figure 1(f)). The pathological diagnosis was a high-grade UC that had invaded the periureteric fat and metastasized to two of four LNs (pT3N2).

At 1.5 months after the surgery, CT revealed no recurrence to the obturator lymph node (Figure 1(i)), but the remaining common iliac LN enlarged to $22.2 \pm 0.8 \mathrm{~mm}$ (Figure $1(\mathrm{j})$ ). Furthermore, the sizes of the two para-aortic LNs significantly increased to $12.7 \pm 0.9 \mathrm{~mm}$ (para-aortic LN \#1, Figure $1(\mathrm{k})$ ) and $17.1 \pm 0.8 \mathrm{~mm}$ (para-aortic LN \#2, Figure 1(1)). The patient underwent 2 additional courses of GC-chemotherapy (GC \#7-8). The common iliac LN and para-aortic LN \#2 shrank, to $15.5 \pm 0.9 \mathrm{~mm}$ (Figure $1(\mathrm{n})$ ) and $7.9 \pm 0.6 \mathrm{~mm}$ (Figure $1(\mathrm{p})$ ), respectively, but the para-aortic LN \#1 did not (Figure 1(o)). After the additional 2 courses of GC-chemotherapy (GC \#9-10), both obturator LN and para-aortic LN \#1 remained within the normal size range (Figures 1(q) and 1(s)), whereas the sizes of the common iliac LN $(23.0 \pm 0.7 \mathrm{~mm}$, Figure $1(\mathrm{r}))$ and para-aortic LN \#2 (11.9 $\pm 0.4 \mathrm{~mm}$, Figure $1(\mathrm{t}))$ increased.

The patient underwent 8 courses of second-line chemotherapy using gemcitabine $\left(1,000 \mathrm{mg} / \mathrm{m}^{2}\right.$, days 1,8 , and 15$)$ and paclitaxel $\left(180 \mathrm{mg} / \mathrm{m}^{2}\right.$, day 1) (GP-chemotherapy \#1-8,
Table S1) [7]. No recurrence was observed at the site of the obturator LN resection (Figure $1(\mathrm{u})$ ), while those of the common iliac LN (5.8 $\pm 0.7 \mathrm{~mm}$, Figure $1(\mathrm{v}))$ and para-aortic LN \#1 (5.8 $\pm 0.6 \mathrm{~mm}$, Figure $1(\mathrm{w})$ ) significantly decreased; however, the size of the para-aortic LN \#2 grew $(21.5 \pm 0.3 \mathrm{~mm}$, Figure $1(\mathrm{x})$ ).

As consolidative therapy for the left common iliac LN and para-aortic LN \#1 as well as salvage therapy for the paraaortic LN \#2, the patient underwent aggressive extra-beam radiotherapy utilizing 10 megavolts photons from a linear accelerator employing a 3-dimensional conformal technique (Figure 2). The final irradiation dose was 60 gray (Gy) in 30 fractions. The patient underwent two patterns of radiotherapy: (A) standard irradiation from the frontal and posterior positions (40 Gy/20 fractions, green-lined area in Figure 2(a)) and (B) boost irradiation from the oblique position (20 Gy/10 fractions, yellow-lined area in Figure 2(b)). During standard irradiation, the gross tumor volume (GTV), identified on CT of the target metastasized lesions in the area, included the para-aortic LN \#2 and the left common iliac LNs (para-aortic LN \#2 and the left common iliac artery/vein, black-dotted lines in Figure 2(a)). Two clinical target volumes (CTVs, i.e., the clinical target of irradiation) were set to the overlapping areas (CTV1 and CTV2 in Figure 2(a)). CTV1 was defined by the aorta (Ao), inferior vena cava (IVC), and left common iliac artery/vein with $7 \mathrm{~mm}$ margins where metastatic cells possibly existed in the lymphatic system, while CTV2 was defined by the GTVs with a $5 \mathrm{~mm}$ margin (pink-circled area in Figure 2(a)). Subsequently, both actual radiation fields, RF1 and RF2, were set based on CTV1 and CTV2, excluding the right kidney, spinal cord, and bowel (green- and yellow-lined areas in Figures 2(a) and 2(b), resp.).

No recurrence was observed at the site of the extracted obturator LN (Figure 1(y)) with no regrowth reported in the two LNs that were irradiated as part of the consolidative therapy (Figures 1(z) and 1(aa)). Remarkably, the para-aortic LN \#2 that presumably contained chemoresistant cancer cells significantly shrunk to normal size after salvage radiotherapy $(5.4 \pm 0.5 \mathrm{~mm}$, Figure $1(\mathrm{ab}))$. The patient is currently free from cancer recurrence/metastasis and severe complications possibly due to aggressive multimodality therapies.

\section{Discussion}

We have described here a case of Stage IV UUT-UC with LN metastases successfully treated with multimodality therapies including (i) RNU with LND after neoadjuvant GCchemotherapy, (ii) salvage GC- and GP-chemotherapy, (iii) consolidative radiotherapy, and (iv) salvage radiotherapy. The ureteral tumor and obturator LN, common iliac LN and paraaortic LN \#1, and para-aortic LN \#2 were cured by (i)-(iii), (ii)-(iii), and (ii)-(iv), respectively. These therapies may therefore be independently effective for heterogeneous cancers with different characteristics in different lymph nodes.

According to the UUT-UC collaboration's report, RNU with LND after neoadjuvant chemotherapy can accomplish pathological N0 in 50\% of UTUC patients with nodal involvement [8], which leads to better overall survival (OS). Therefore, in the present case, we attempted neoadjuvant 


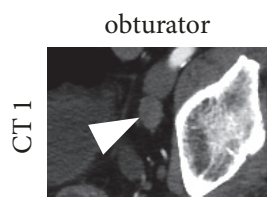

(a)

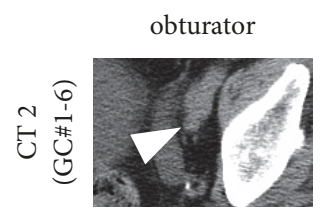

(e)

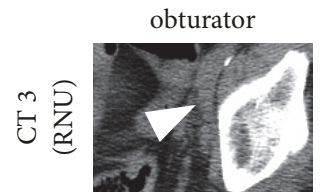

(i)

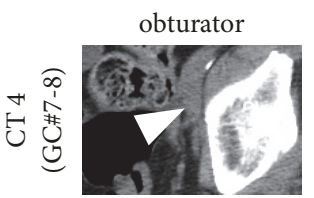

(m)

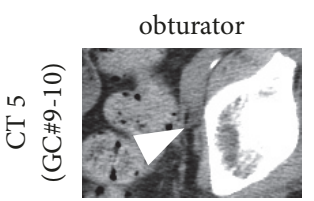

(q)

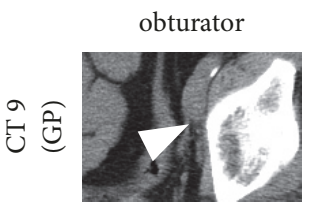

(u)

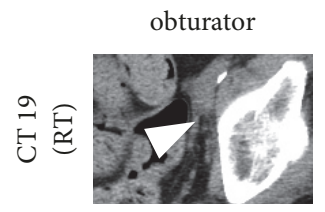

(y)

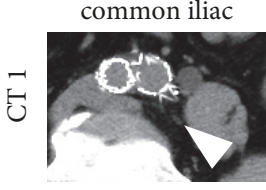

(b)

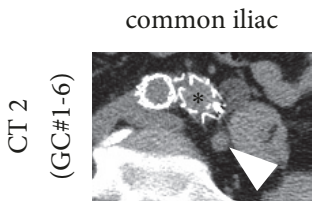

(f)

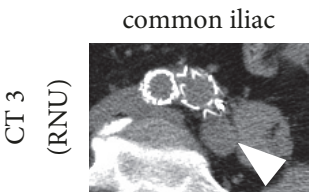

(j)

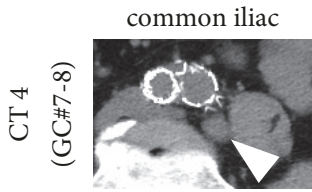

(n)

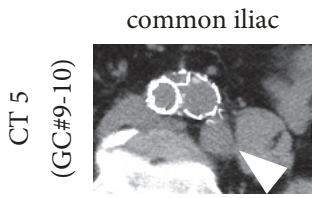

(r)

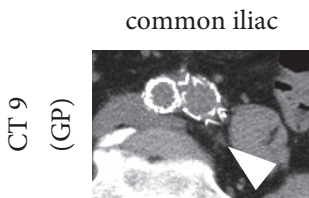

(v)

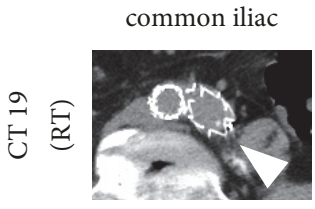

(z)

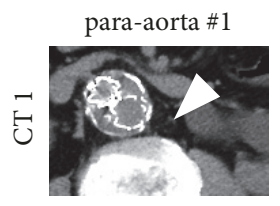

(c)

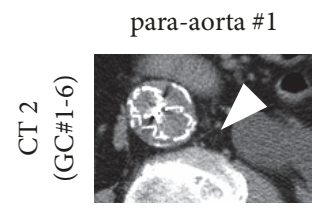

(g)

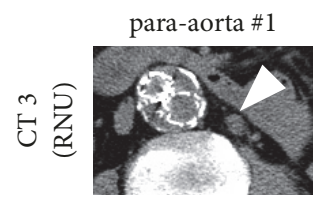

(k)

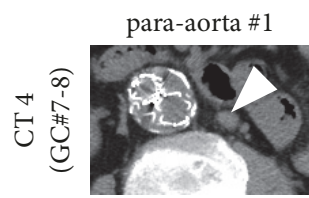

(o)

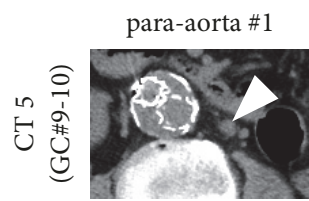

(s)

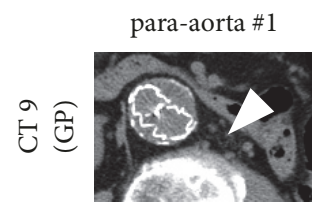

(w)

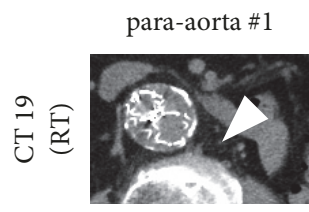

(aa)

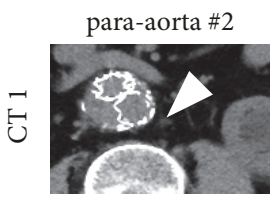

(d)

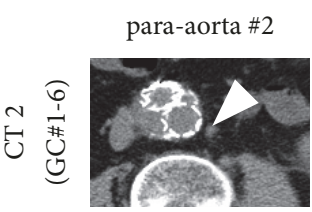

(h)

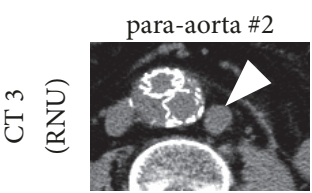

(l)

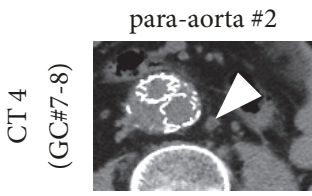

(p)

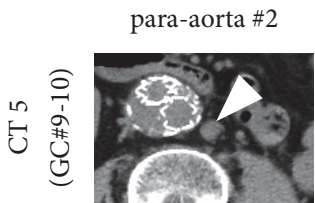

(t)

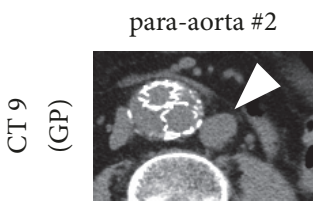

(x)

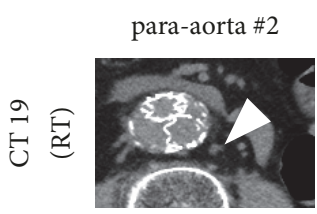

(ab)

Figure 1: Selected CT images of the lymph nodes for every treatment modality. (a-d), (e-h), (i-l), (m-p), (q-t), (u-x), and ( $\mathrm{y}-\mathrm{ab})$ represent CT scans \#1, \#2, \#3, \#4, \#5, \#9, and \#19 in supplemental Figure 1, respectively. Arrowheads in the 1st to 4th columns show the obturator lymph nodes, common iliac lymph nodes, para-aortic lymph node \#1, and para-aortic lymph node \#2, respectively. CT: computed tomography, GC: chemotherapy using gemcitabine and cisplatin, RNU: radical nephroureterectomy, GP: chemotherapy using gemcitabine and paclitaxel, and RT: radiotherapy.

chemotherapy and curative LND based on possible anatomical lymphatic drainage from the lower ureter: obturator LNs (Figures 1(a) and 1(e)), a part of left common iliac LNs (Figures 1(b) and 1(f)), and internal and external iliac
LNs (data not shown) $[5,6]$. The metastasized obturator LN may have been cured only by LND with neoadjuvant GCchemotherapy because the area was not irradiated (Figures $1(\mathrm{i}), 1(\mathrm{~m})$, and $1(\mathrm{q}))$. This result supports the UUT-UC 

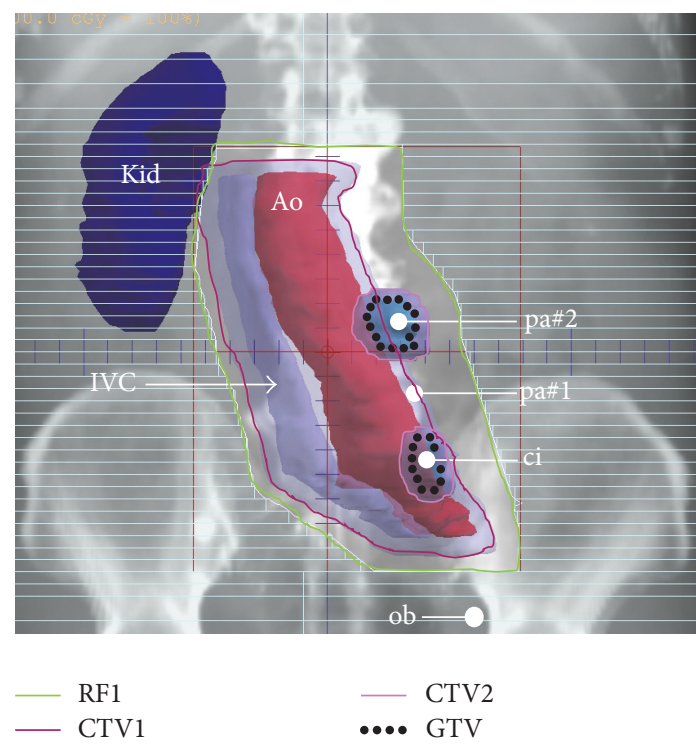

(a) Standard

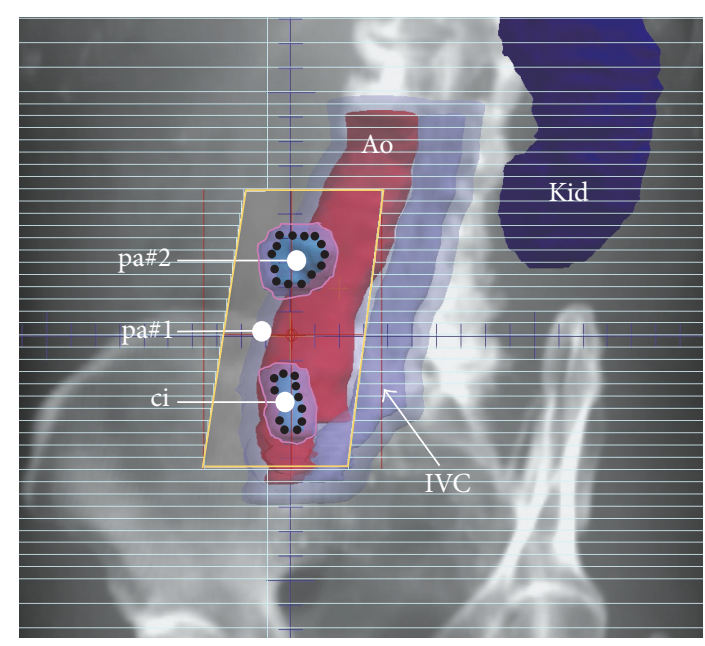

$\begin{array}{ll}- & \text { RF2 } \\ - & \text { CTV2 } \\ \cdots & \text { GTV }\end{array}$

(b) Boost

FIGURE 2: Irradiation conditions for the lymph nodes. (a) Frontal view of CT image reconstruction; RF1 was set based on CTV1 and 2 as standard radiation. (b) Left anterior oblique view of CT image reconstruction; RF2 was set based on CTV2 as a radiation boost. CT: computed tomography, RF: radiation field, GTV: gross tumor volume, CTV: clinical target volume, Ao: aorta, IVC: inferior vena cava, Kid: right kidney, ob: obturator lymph node, ci: left common iliac lymph node, and pa: para-aortic lymph node.

collaboration's report mentioned above, and we are convinced that complete resection after neoadjuvant chemotherapy can cure metastatic UTUC.

However, the unremoved common iliac LN and paraaortic LNs increased in size early after the surgery. Matin et al. have reported that approximately $30 \%$ of LN involvement in lower UUT-UC unexpectedly spread to the para-aortic region [9], suggesting that the extent of LND in this case was insufficient. Further extended LND may be considered when conducting RNU as the common iliac LN was swollen.

Using data of bladder cancer, methods of combination chemotherapies are applied for UUT-UC because of its rarity [10]. As first line treatment, platinum-based combination chemotherapies including GC are generally used for advanced UC [10]. As second line treatment, GP-chemotherapy achieves an overall response rate of 30 to $70 \%$ [7]. In cisplatin-ineligible UC, taxanes with gemcitabine may have similar additional effects compared to carboplatin with gemcitabine (53\% versus $45 \%$ ) [11]. GC with paclitaxel may improve OS without increasing side effects [12]. Moreover, regimens with taxanes had a tolerable toxicity profile, even if patients have renal dysfunction post-RNU. In fact, complete response was obtained by treatment with GP [13]. In our case, the sizes of the two LNs (common iliac (Figures 1(r) and 1(v)) and para-aortic LN \#1 (Figures 1(s) and 1(w)), resp.), with resistance to platinum-based chemotherapy, were also significantly reduced by GP-chemotherapy as a second-line treatment. Further compilation of cases cured by GP is needed to substantiate this superior UUT-UC survival outcome.

In patients with UUT-UC, bladder recurrence and distant metastases are the most frequently reported patterns, whereas isolated lymph node recurrence(s) is rare [14]. According to previous reports, adjuvant radiotherapy does not control lymph node metastases or improve OS [15-17]. However, Fan et al. showed that the group of UUT-UC patients treated with a prescribed dose of over $50 \mathrm{~Gy}$ as salvage radiotherapy had significantly longer progression-free survival and OS than the group of patients treated with a low dose; they emphasized that a high prescribed dose ( $\geq 50 \mathrm{~Gy})$ was required in order to achieve curative outcomes [18]. Meanwhile, a small cohort study about consolidative radiotherapy reported the long-term clinical benefit of consolidative radiotherapy after chemotherapy (the prescribed dose ranged from 50 to $60 \mathrm{~Gy}$ ) for nodal recurrences of UUT-UC treated with RNU [19]. In our case, the size of the para-aortic LN \#2 that showed chemoresistance may have been significantly reduced by the total dose of $60 \mathrm{~Gy}$ as salvage radiotherapy (Figures 1(x), 1(ab), and 2). Additionally, both the common iliac LN and para-aortic LN \#1 were also irradiated with consolidative radiotherapy after chemotherapy and are still reducing in size (Figures $1(\mathrm{v})$ and $1(\mathrm{w}), 1(\mathrm{z})$ and $1(\mathrm{aa})$ ). As a result, the patient has shown no evidence of recurrence and metastasis or any severe complications associated with radiotherapy for approximately 4 years from the initial diagnosis and for 18 months after radiotherapy.

\section{Conclusions}

Radiotherapy for LN metastasis in advanced UUT-UC has not yet been recommended. Our case suggests that salvage or consolidative radiotherapy combined with LND and perioperative chemotherapies may have clinical benefits in selected 
cases of advanced UUT-UC with LN metastasis. Further compilation of cases is needed to select appropriate patients, as well as standardizing radiotherapy methods, including range and dosage.

\section{Abbreviations}

$\begin{array}{ll}\text { UUT-UC: } & \begin{array}{l}\text { Upper urinary tract urothelial } \\ \text { carcinoma }\end{array} \\ \text { LN: } & \text { Lymph node } \\ \text { RNU: } & \text { Radical nephroureterectomy } \\ \text { RT: } & \text { Radiotherapy } \\ \text { CT: } & \text { Computed tomography } \\ \text { UC: } & \text { Urothelial carcinoma } \\ \text { GC-chemotherapy: } & \text { Gemcitabine and cisplatin } \\ & \text { chemotherapy } \\ \text { LND: } & \text { Lymph node dissection } \\ \text { GP-chemotherapy: } & \text { Gemcitabine and paclitaxel } \\ & \text { chemotherapy } \\ \text { GTV: } & \text { Gross tumor volume } \\ \text { CTV: } & \text { Clinical target volume } \\ \text { RF: } & \text { Radiation field. }\end{array}$

\section{Consent}

Written informed consent was obtained from the patient for publication of this case report and any accompanying images. A copy of the written consent is available for review by the Editor-in-Chief of this journal.

\section{Disclosure}

Hideyuki Kondo and Suguru Shirotake are co-first authors.

\section{Conflicts of Interest}

The authors have read and understood Current Oncology's policy on disclosing conflicts of interest and declare that they have none.

\section{Authors' Contributions}

Hideyuki Kondo, Takashi Okabe, and Suguru Shirotake performed acquisition and interpretation of data. Suguru Shirotake and Koshiro Nishimoto drafted the manuscript. Soichi Makino, who is a radiologist, managed radiotherapy in this case. Koshiro Nishimoto and Masafumi Oyama revised this manuscript critically for important intellectual content. All authors read and approved the version to be published.

\section{Acknowledgments}

The authors thank Akari Komatsuda (AK) for acquisition of clinical data.

\section{Supplementary Materials}

Supplementary 1. Figure S1: magnetic resonance imaging of the lower left ureteral tumor. The ureteral tumor was $21 \mathrm{~mm}$ in diameter at the initial diagnosis (A). The tumor temporarily reduced in size after 3 courses of GC-chemotherapy (B). The tumor reenlarged after an additional 3 courses of GCchemotherapy (C). The asterisks and red and light blue arrowheads indicate the bilateral femoral heads, ureteral tumor, and hydroureter, respectively.

Supplementary 2. Figure S2: CT imaging of the lymph nodes. Total CT imaging of the lymph nodes: obturator, common iliac, and para-aortic lymph nodes. Repetitive CT imaging was performed 19 times since initial diagnosis, to evaluate recurrence and metastasis. The white arrowheads indicate the lymph nodes.

Supplementary 3. Table S1: lymph nodes of clinical course of the case.

\section{References}

[1] M. Roupret et al., "European association of urology guidelines on upper urinary tract urothelial cell carcinoma: 2015 update," European Urology, vol. 68, no. 5, pp. 868-879, 2015.

[2] A. J. Wein and et al., Campbell-Walsh Urology, Elsevier Health Sciences, 2011.

[3] S. F. Matin, V. Margulis, A. Kamat et al., "Incidence of downstaging and complete remission after neoadjuvant chemotherapy for high-risk upper tract transitional cell carcinoma," Cancer, vol. 116 , no. 13, pp. 3127-3134, 2010.

[4] H. von der Maase, L. Sengelov, J. T. Roberts et al., "Long-term survival results of a randomized trial comparing gemcitabine plus cisplatin, with methotrexate, vinblastine, doxorubicin, plus cisplatin in patients with bladder cancer," Journal of Clinical Oncology, vol. 23, no. 21, pp. 4602-4608, 2005.

[5] T. Kondo, H. Nakazawa, F. Ito, Y. Hashimoto, H. Toma, and K. Tanabe, "Impact of the Extent of Regional Lymphadenectomy on the Survival of Patients With Urothelial Carcinoma of the Upper Urinary Tract," The Journal of Urology, vol. 178, no. 4, pp. 1212-1217, 2007.

[6] T. Kondo and K. Tanabe, "Role of lymphadenectomy in the management of urothelial carcinoma of the bladder and the upper urinary tract," International Journal of Urology, vol. 19, no. 8, pp. 710-721, 2012.

[7] T. Suyama, T. Ueda, S. Fukasawa et al., "Combination of gemcitabine and paclitaxel as second-line chemotherapy for advanced urothelial carcinoma," Japanese Journal of Clinical Onco$\log y$, vol. 39, no. 4, pp. 244-250, 2009.

[8] H. Kitamura, "Upper urinary tract urothelial carcinoma with loco-regional nodal metastases: Insights from the Upper Tract Urothelial Carcinoma Collaboration," BJU International, vol. 110, no. 11, p. E435, 2012.

[9] S. F. Matin, J. P. Sfakianos, P. N. Espiritu, J. A. Coleman, and P. E. Spiess, "Patterns of lymphatic metastases in upper tract urothelial carcinoma and proposed dissection templates," The Journal of Urology, vol. 194, no. 6, pp. 1567-1574, 2015.

[10] S. Shirotake et al., "Impact of an adjuvant chemotherapeutic regimen on the clinical outcome in high-risk patients with upper tract urothelial carcinoma," The Journal of Urology, vol. 193, no. 4, pp. 1122-1128, 2015.

[11] A. Necchi, G. R. Pond, D. Raggi et al., "Efficacy and Safety of Gemcitabine Plus Either Taxane or Carboplatin in the FirstLine Setting of Metastatic Urothelial Carcinoma: A Systematic 
Review and Meta-Analysis," Clinical Genitourinary Cancer, vol. 15, no. 1, pp. 23-30.e2, 2017.

[12] P. Giannatempo, G. R. Pond, G. Sonpavde et al., "The impact of adding taxanes to gemcitabine and platinum chemotherapy for the first-line therapy of advanced or metastatic urothelial cancer: A systematic review and meta-analysis," European Urology, vol. 69, no. 4, pp. 624-633, 2016.

[13] K. Kanai, E. Kikuchi, T. Ohigashi et al., "Gemcitabine and paclitaxel chemotherapy for advanced urothelial carcinoma in patients who have received prior cisplatin-based chemotherapy," International Journal of Clinical Oncology, vol. 13, no. 6, pp. 510514, 2008 .

[14] V. Margulis, S. F. Shariat, S. F. Matin et al., "Outcomes of radical nephroureterectomy: A series from the upper tract urothelial carcinoma collaboration," Cancer, vol. 115, no. 6, pp. 1224-1233, 2009.

[15] M. C. Hall, J. S. Womack, C. G. Roehrborn, T. Carmody, and A. I. Sagalowsky, "Advanced transitional cell carcinoma of the upper urinary tract: Patterns of failure, survival and impact of postoperative adjuvant radiotherapy," The Journal of Urology, vol. 160, no. 3 I, pp. 703-706, 1998.

[16] S. Ananthula, P. Parajuli, and F. A. Behery, "Adjuvant radiotherapy for stage III/IV urothelial carcinoma of the upper tract," Anticancer Reseach, vol. 34, no. 1, pp. 333-338, 2014.

[17] A. W. Hahn, S. Giri, R. Pathak, V. R. Bhatt, and M. G. Martin, "Effect of adjuvant radiotherapy on survival in patients with locoregional urothelial malignancies of the upper urinary tract," Anticancer Reseach, vol. 36, no. 8, pp. 4051-4055, 2016.

[18] K. H. Fan et al., "Adjuvant and salvage radiotherapy for urothelial cell carcinoma of the upper urinary tract: experience in a single institution," Chang Gung Medical Journal, vol. 35, no. 3, pp. 247-254, 2012.

[19] M. Y. Al-Marrawi, H. B. Mackley, S. Ali et al., "Consolidation With Radiation or Concurrent Chemo-Radiation After Chemotherapy Results in Durable Complete Remissions of Isolated Nodal Recurrences of Urothelial Cancer: A Case Series and Review," Clinical Genitourinary Cancer, vol. 14, no. 4, pp. e393e399, 2016. 


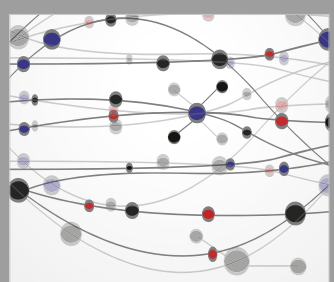

The Scientific World Journal
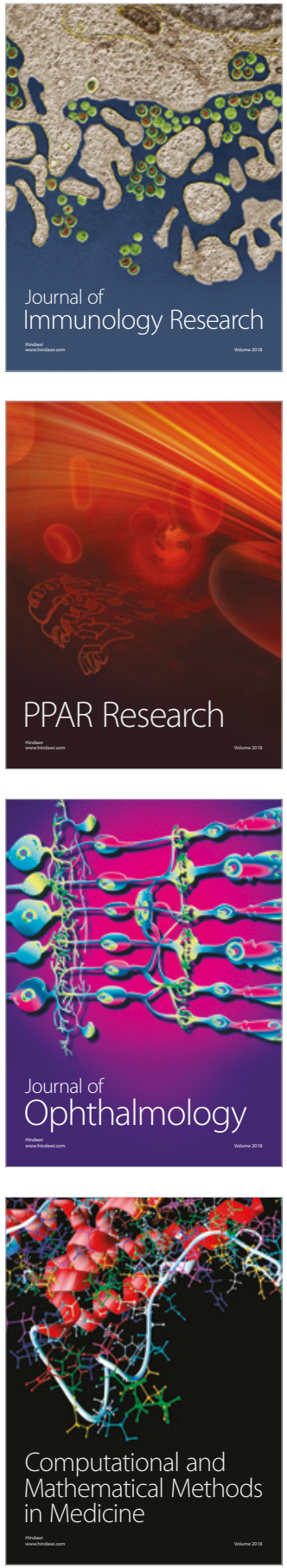

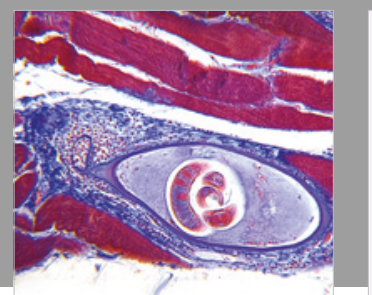

Gastroenterology Research and Practice

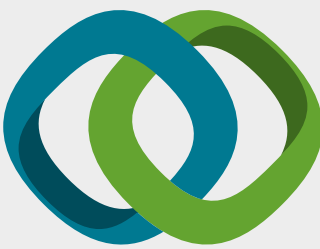

\section{Hindawi}

Submit your manuscripts at

www.hindawi.com
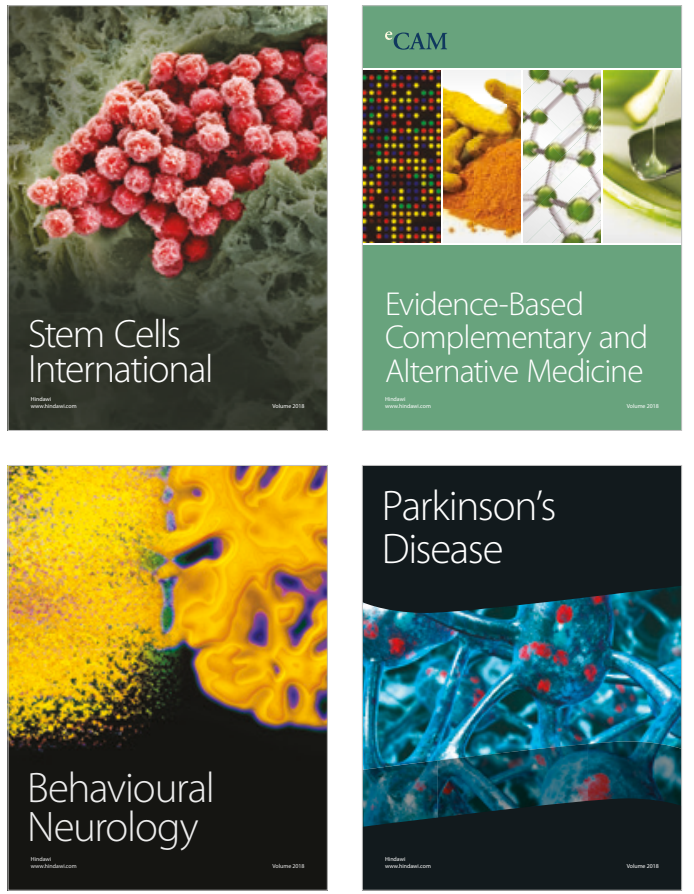

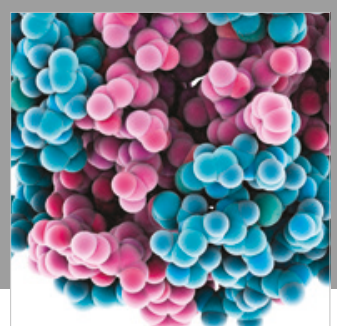

ournal of

Diabetes Research

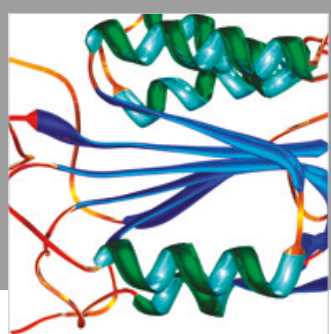

Disease Markers
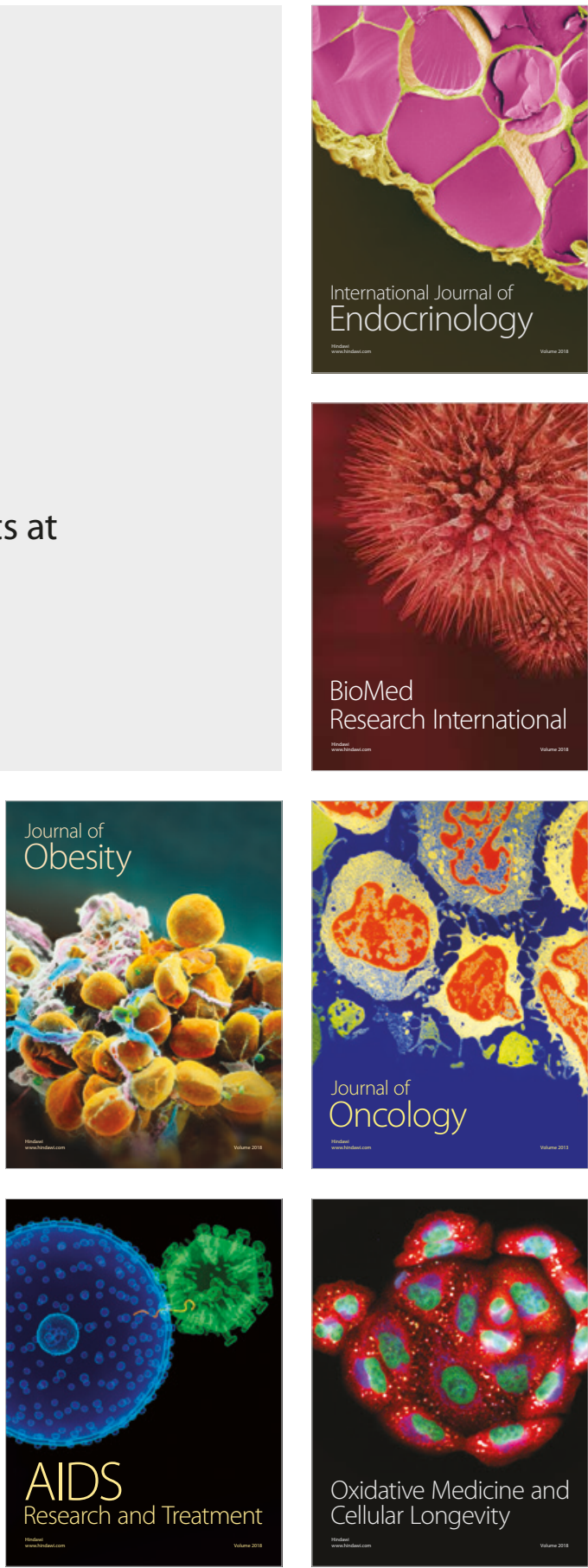